\title{
Characteristics and impact of exercise- induced laryngeal obstruction: an international perspective
}

\author{
Emil S. Walsted (i) ${ }^{1,2}$, Bamidele Famokunwa ${ }^{3}$, Louise Andersen ${ }^{4}$, \\ Sune L. Rubak ${ }^{4}$, Frederik Buchvald ${ }^{5}$, Lars Pedersen (1) ${ }^{1}$, James Dodd (1) ${ }^{3}$, \\ Vibeke Backer ${ }^{6}$, Kim G. Nielsen ${ }^{5,7}$, Andrew Getzin ${ }^{8}$ and James H. Hull (1) ${ }^{2}$
}

\begin{abstract}
Affiliations: ${ }^{1}$ Dept of Respiratory Medicine, Bispebjerg Hospital, Copenhagen, Denmark. ${ }^{2}$ Dept of Respiratory Medicine, Royal Brompton Hospital, London, UK. ${ }^{3}$ Academic Respiratory Unit, University of Bristol, Southmead Hospital, Bristol, UK. " Dept of Pediatrics and Adolescent Medicine, Aarhus University Hospital Skejby, Aarhus, Denmark. ${ }^{5}$ Pediatric Pulmonary Service, Dept of Pediatrics and Adolescent Medicine, Rigshospitalet, Denmark. ${ }^{6}$ Dept of ENT and Centre for Physical Activity Research, Rigshospitalet and Copenhagen University, Copenhagen, Denmark. ${ }^{7}$ Dept of Clinical Medicine, University of Copenhagen, Copenhagen, Denmark. ${ }^{8}$ Cayuga Medical Center, Ithaca, NY, USA.
\end{abstract}

Correspondence: Emil S. Walsted, Respiratory Research Unit, Department of Respiratory Medicine, Bispebjerg Hospital, Bispebjerg Bakke 23, 2400 Copenhagen NV, Denmark. E-mail: emilwalstedadadlnet.dk

\section{ABSTRACT}

Background: Exercise-induced laryngeal obstruction (EILO) is a common cause of exertional breathlessness and wheeze yet is frequently misdiagnosed as asthma. Insight regarding the demographic characteristics, laryngeal abnormalities and impact of EILO is currently limited, with data only available from individual centre reports. The aim of this work was to provide a broader perspective from a collaboration between multiple international expert centres.

Methods: Five geographically distinct clinical paediatric and adult centres (3 Denmark, 1 UK, 1 USA) with an expertise in assessing unexplained exertional breathlessness completed database entry of key characteristic features for all cases referred with suspected EILO over a 5-year period. All included cases completed clinical asthma workup and continuous laryngoscopy during exercise (CLE) testing for EILO.

Results: Data were available for 1007 individuals $(n=713$ female $(71 \%)$ ) with a median (range) age of 24 (8-76) years, and of these $586(58 \%)$ were diagnosed with EILO. In all centres, EILO was frequently misdiagnosed as asthma; on average there was a 2-year delay to diagnosis of EILO, and current asthma medication was discontinued in $20 \%$. Collapse at the supraglottic level was seen in $60 \%$, whereas vocal cord dysfunction (VCD) was only detected/visualised in $18 \%$. Nearly half (45\%) of individuals with EILO were active participants in recreational-level sports, suggesting that EILO is not simply confined to competitive/elite athletes.

Conclusion: Our findings indicate that key clinical characteristics and the impact of EILO/VCD are similar in globally distinct regions, facilitating improved awareness of this condition to enhance recognition and avoid erroneous asthma treatment.

@ERSpublications

Exercise-induced laryngeal obstruction is a prevalent cause of exertional breathlessness and wheeze. In this international multicentre collaboration, 1007 patients waited, on average, 2 years for diagnosis and $\mathbf{2 0 \%}$ were mistreated as asthmatic. https://bit.ly/3auXpcp

Cite this article as: Walsted ES, Famokunwa B, Andersen L, et al. Characteristics and impact of exercise-induced laryngeal obstruction: an international perspective. ERJ Open Res 2021; 7: 001952021 [https://doi.org/10.1183/23120541.00195-2021]. 


\section{Introduction}

Exercise-induced laryngeal obstruction (EILO) describes a phenomenon in which the laryngeal structures temporarily close during vigorous exercise, resulting in exertional breathlessness, cough and wheeze [1]. This condition remains frequently overlooked and misdiagnosed as asthma $[2,3]$, and thus individuals with EILO often fail to receive appropriate management and at the same time are typically inappropriately prescribed unnecessary asthma therapy [4].

To date, the published literature describing the characteristics of individuals with EILO arises almost exclusively from single-centre reports, originating from a small number of highly specialised centres located in Scandinavian countries [2]. Data from this geographical region indicate that EILO is common, affecting $\sim 5-7 \%$ of adolescents $[3,5]$ and up to a quarter of athletes presenting with exertional "asthma-type" symptoms despite no objective evidence of asthma [4]. These reports also indicate that EILO has a female preponderance and a peak age of onset in teenage years. Importantly, the published analyses also highlight a consistent and significant delay in time to diagnosis, often taking many years from symptom onset and associated with exercise avoidance [6, 7] and heightened anxiety [8].

Although somewhat informative, single-centre reports are limited by local referral bias and practice, exercise engagement within the local community, and care provision and accessibility within a given healthcare system. They can thus only be expected to provide a relatively limited perspective on the true characteristics of EILO. Indeed, it remains the case that, outside of the Scandinavian region, there is very limited insight regarding the characteristics of this condition. It is also not clear how different centres approach treatment.

The aim of this study was to compile a geographically broad and regionally diverse dataset, drawn from several adult and paediatric respiratory centres, to provide a more general perspective on the typical characteristics of individuals undergoing assessment for suspected EILO. We recruited centres from the UK and US, to provide comparison data with adult and paediatric centres in Scandinavia. We aimed to evaluate and describe four key areas concerning EILO, including: 1) demographics, 2) time to diagnosis, 3) findings on testing and 4) approach to treatment. A key aim was to evaluate time to diagnosis and (over-) use of asthma medication with an over-arching aim of increasing understanding of EILO, to provide increased clinical insight and awareness of this condition.

\section{Methods}

\section{Design and study population}

Five clinical centres (Royal Brompton Hospital, London, UK; Bispebjerg Hospital and Rigshospitalet, Region H, Copenhagen, Denmark; Aarhus University Hospital Skejby, Aarhus, Denmark; and Cayuga Medical Center, New York State, USA) provided data in a retrospective observational evaluation of individuals referred with possible EILO (i.e. unexplained exertional dyspnoea or symptoms highly suggestive of laryngeal closure on exertion such as: exertional dyspnoea, audible inspiratory breathing sounds, localisation of symptoms to the throat/neck or asthma-like symptoms previously unexplained by previous objective testing for asthma), between January 1, 2014 and December 31, 2018.

Each centre extracted their own data to address key characteristics and basic anthropometric information. Each centre obtained local service evaluation/ethical approval to complete analysis of their dataset in a fully anonymised form, and data were not shared between centres. This undertaking was approved by internal review boards at Cayuga Medical Center (no. 0718AG), Aarhus University Hospital (no. 1-16-02-326-15), Bispebjerg Hospital and Rigshospitalet, Region H (no. WZ18044879) and Royal Brompton Hospital (CIRIS 002771).

\section{Diagnosis of EILO}

All centres utilise the gold standard test for the diagnosis of EILO, namely the continuous laryngoscopy during exercise (CLE) test. Briefly, this test requires placement and fixation of a flexible laryngoscope, to allow continuous, uninterrupted acquisition of laryngeal movement via video capture [9]. Evidence of EILO was taken, using the most widely utilised grading system (with a score of $\geqslant 2$ [10] as EILO), also describing the predominant site of closure, at either the supraglottic, glottic level or combined closure (figure 1). Individuals who were not classifiable by this grade scoring scheme (e.g. anatomical variations, laryngeal lesions, etc.) were coded as "other".

\section{Asthma and other comorbidities}

A diagnosis of asthma/no asthma was made by the clinical team following completion of the diagnostic workup, i.e. considering all available diagnostic information, including symptoms, medical history and objective testing. All centres have expertise in the assessment of asthma and utilise bronchial provocation testing, based on guideline recommendations. Individuals with asthma and exercise-induced 

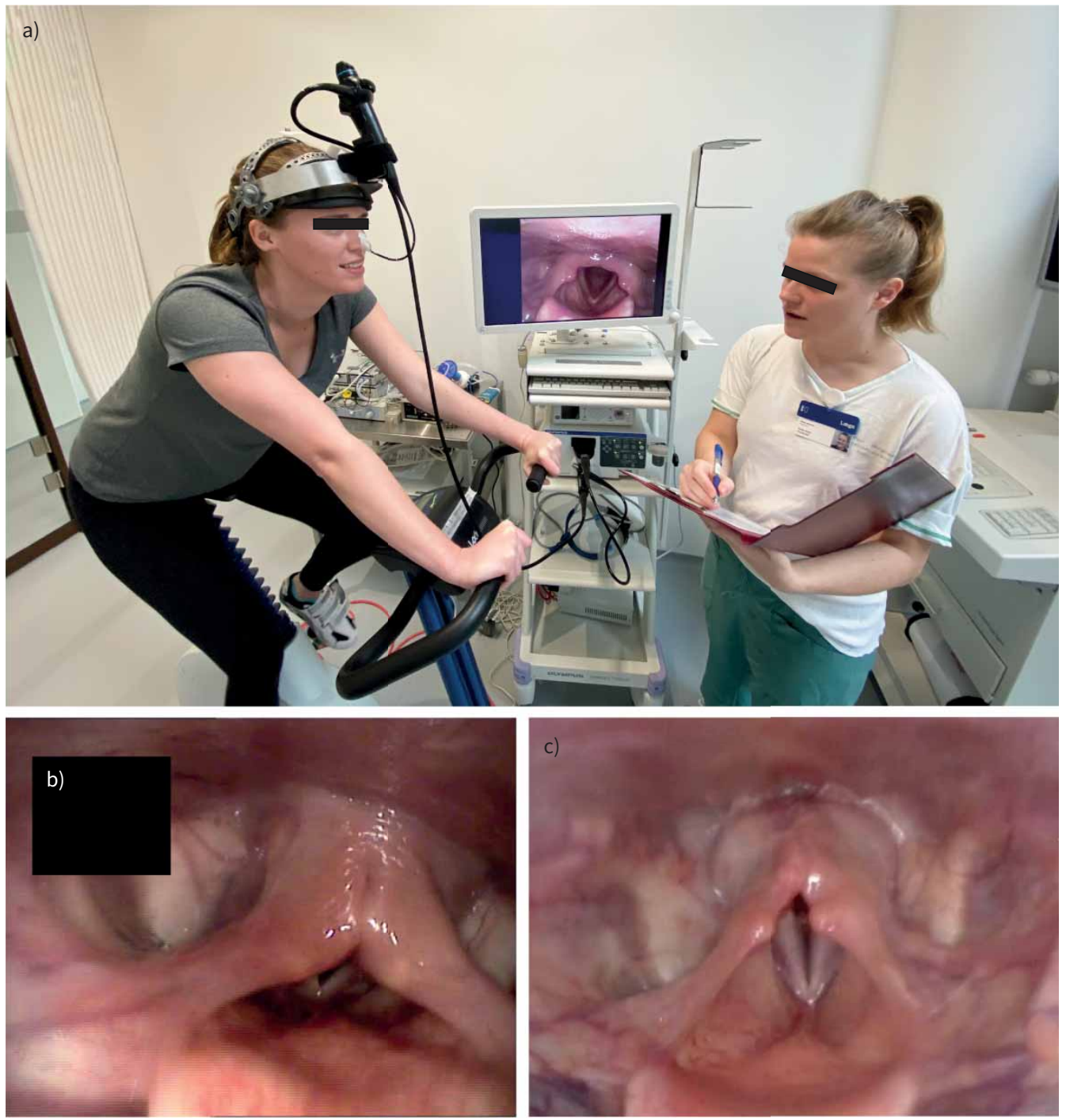

FIGURE 1 a) Continuous laryngoscopy exercise testing setup: the test subject exercises on a stationary ergometer bicycle with a laryngoscope in situ, fastened to specialist headgear. The subject performs an incremental-load exercise test to volitional maximum, while laryngoscopic recordings are saved for subsequent review. Note the laryngoscopic image on-screen showing a healthy larynx anatomy during inspiration. b) Laryngoscopic image (anonymised), taken during inspiration of an individual with supraglottic exercise-induced laryngeal obstruction (EILO), i.e. medial collapse of the arytenoid and aryepiglottic mucosa. The vocal folds are not affected. c) Laryngoscopic image, taken during inspiration of an individual with glottic EILO, i.e. a medial movement of the vocal folds.

bronchoconstriction (EIB) and individuals with EIB alone were all grouped as "asthma". Other comorbidities were recorded.

Time to diagnosis and approach to treatment

Information on the self-reported duration of an individual's respiratory symptoms (recorded in months) and current and previous asthma medications (coded as yes/no) was recorded. Individuals prescribed asthma medication at the time of assessment but with no historical or current objective evidence of asthma were classified as having received erroneous/unnecessary asthma therapy.

We categorised EILO intervention into six general types of treatment: respiratory physiotherapy, biofeedback, inspiratory muscle training, exercise, speech and language therapy (SLT), and surgical intervention with supraglottoplasty.

\section{Analysis and statistics}

Each centre supplied summary descriptive statistics for their pooled data, including mean and standard deviations, or pooled median and range for continuous data, and count and percentages for categorical data. 
Categorical variables were compared using $\chi^{2}$ or, if appropriate, Fisher's exact test; continuous variables were compared using the independent t-test or the Mann-Whitney U-test, depending on distribution. Associations between continuous variables were quantified by simple correlation coefficients (Pearson or Spearman as appropriate). A p-value of $<0.05$ was considered statistically significant.

Analysis and figures were produced using $\mathrm{R}$ version 3.5.0 ( $\mathrm{R}$ Core Team 2019) including the package metamedian [11] and Python programming language version 3.6 (Python Software Foundation, www. python.org).

\section{Results}

\section{Demographic characteristics}

In total, 1008 individuals (71\% female, age range 8-76 years) were CLE tested over the 5-year period, and $586(58 \%)$ were confirmed to have EILO (table 1). In the paediatric centres, the median age was 13 years, with a female predominance (table 1), and similar age at diagnosis was apparent between males and females. The most frequent age of EILO onset was in adolescence (figure 2), and half of the overall EILO population were below age 24 years; however, $15 \%$ were $>30$ years of age at the onset of symptoms. Subjects with EILO were on average younger (median age 21) than those without EILO (median age 27.5 years; $\mathrm{p}<0.001$ ) and a higher proportion of individuals were female in the EILO group (76\% versus $64 \%$, respectively; $\mathrm{p}<0.001$ ) (figure 3 ), while there was no difference in body mass index between the groups (table 2). General demographic characteristics and diagnoses are shown in table 1.

Overall, $45 \%$ of the total referral population were taking part in recreational-level sport (i.e. not elite or competitive), although this differed between centres (table 1); a higher proportion of individuals with EILO were athletic to a competitive level $(\mathrm{p}<0.001)$ (table 2$)$. The predominant sport performed by individuals with EILO was endurance-intensity, e.g. running, swimming and cycling (table 3).

\section{Asthma, EILO and other comorbidities}

The most prevalent diagnoses in this population were EILO (58\% overall, $46-80 \%$ in individual centres) and asthma (39\% overall, $14-52 \%$ in individual centres). Comorbid asthma and EILO was reported in $36 \%$ of individuals and thus was more frequent than in CLE-tested individuals overall (21\%, see tables 1 and 2) $(\mathrm{p}=0.02)$. Approximately one-third of individuals with EILO were currently or had previously been prescribed asthma inhalers, in the absence of confirmatory evidence of asthma (table 3 ).

Other comorbid conditions in those with confirmed EILO included allergy (10\%), rhinitis (7\%), breathing pattern disorder (5\%) and reflux (3\%). Psychiatric/psychological comorbidity was not reported as a comorbidity, with the exception of Rigshospitalet where it was documented in $5 \%$ of children and young adults.

\section{TABLE 1 Demographics of individuals with continuous laryngoscopy during exercise}

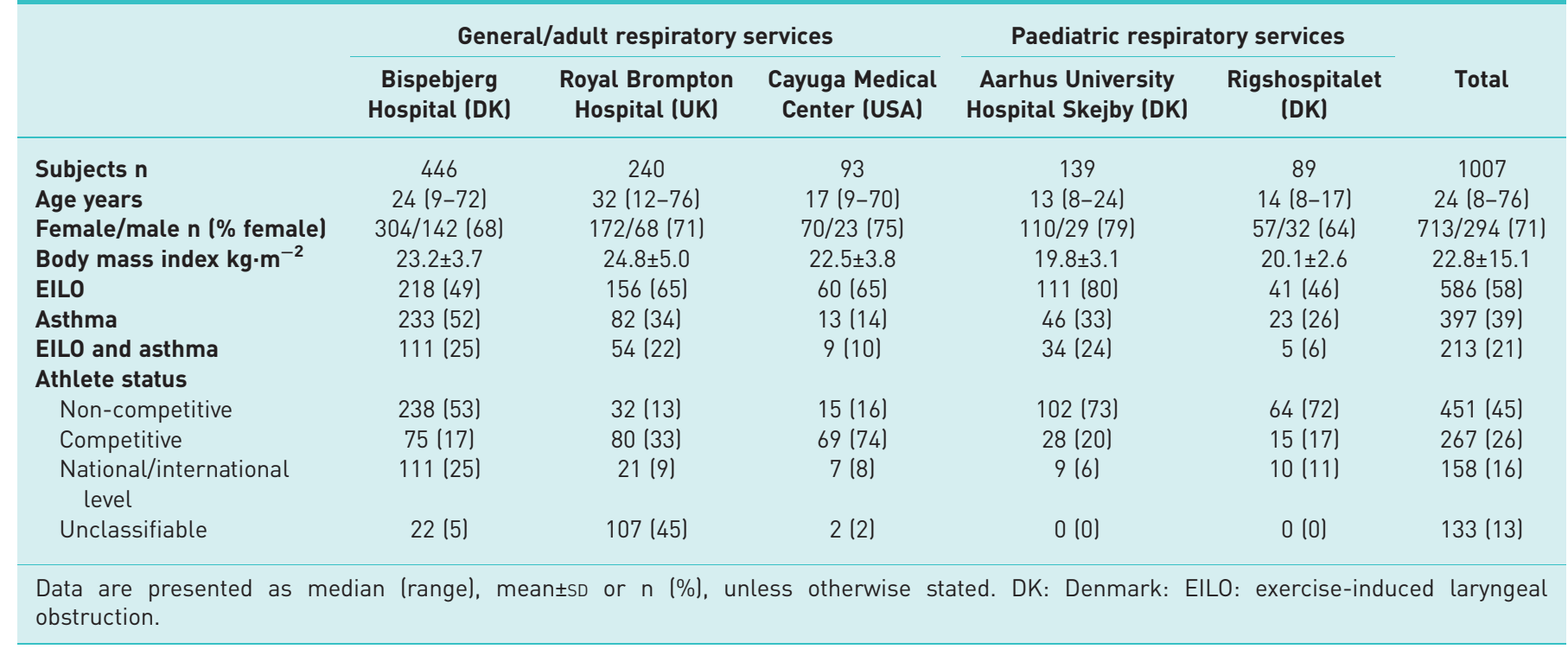



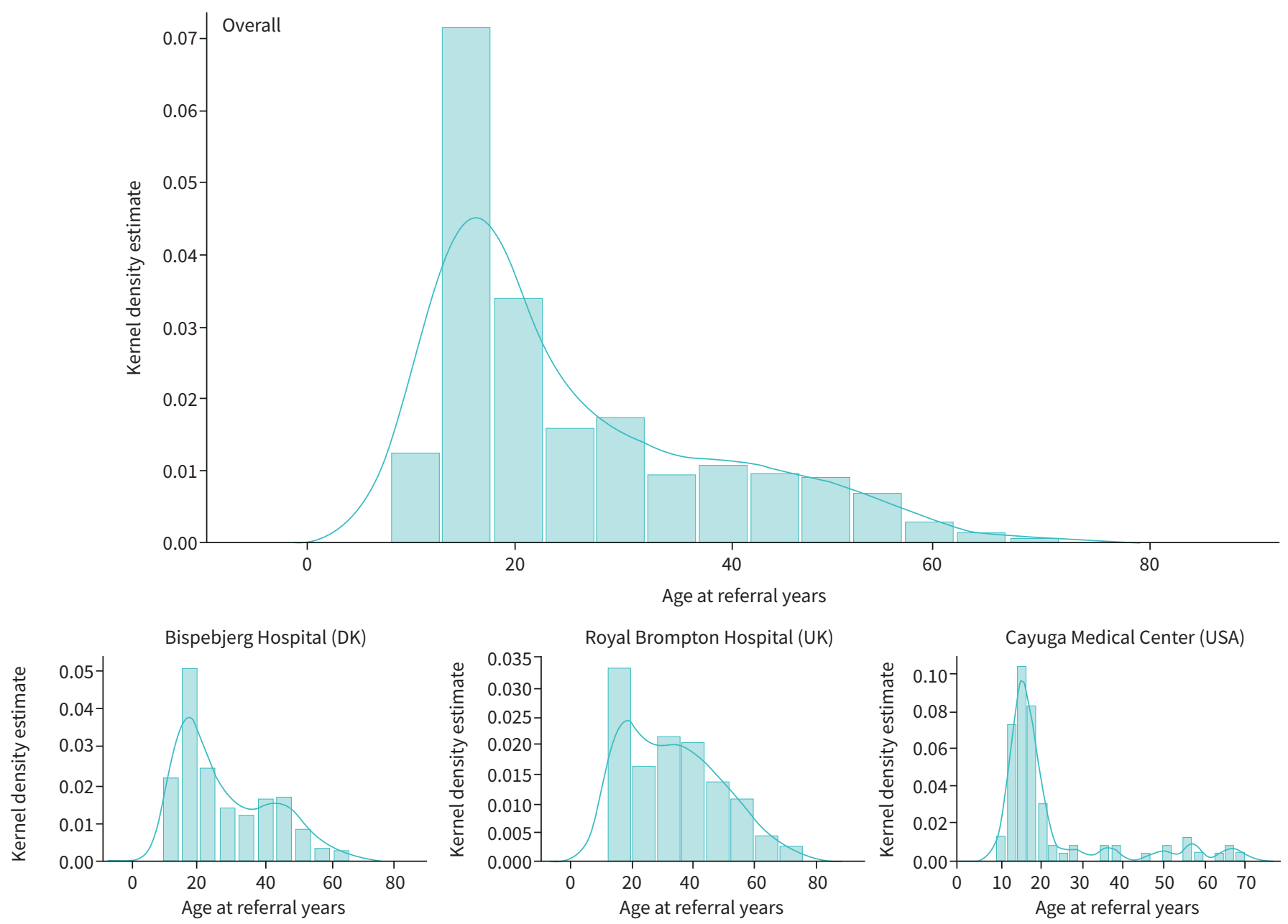

Aarhus University Hospital Skejby (DK)
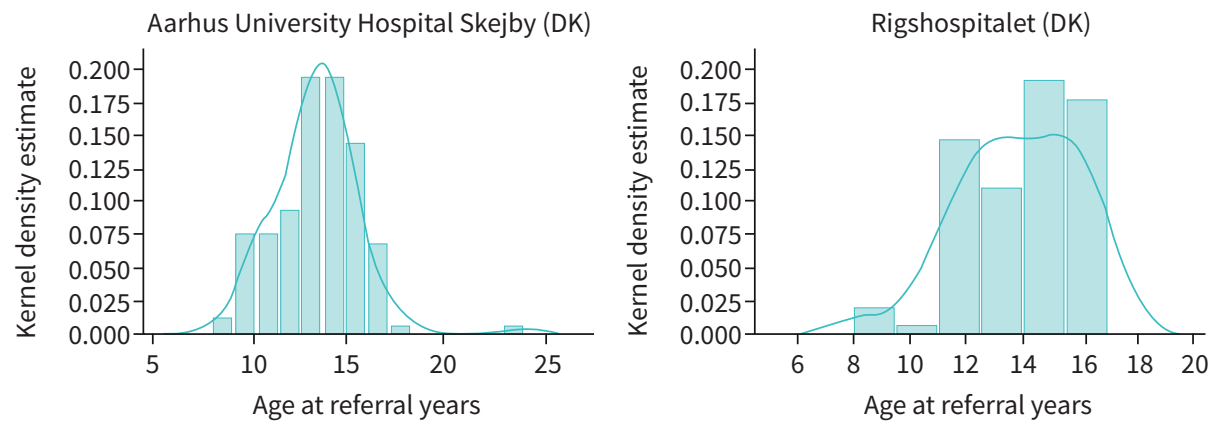

FIGURE 2 Age distribution in individuals with exercise-induced laryngeal obstruction, overall and by centre. Top row: overall; middle row: adult/ mixed respiratory centres; bottom row: paediatric respiratory centres. DK: Denmark.

\section{CLE findings}

The most frequent finding on CLE testing was supraglottic-type EILO, i.e. closure at the level of the arytenoids (60\%), and in some centres accounted for nearly $80 \%$ of cases, while glottic predominant EILO (i.e. often termed "exercise-induced vocal cord dysfunction") was seen in only $18 \%$ of individuals; this was true for all centres with the exception of Cayuga Medical Center where combined supraglottic and glottic EILO was predominant (65\%) (figure 4). Combined glottic and supraglottic EILO was seen in 19\% and unclassifiable subtypes in $3 \%$ of individuals with confirmed EILO.

Referral pathway and treatment approach

Individuals with EILO reported having had exercise-related respiratory symptoms for a median of 2 years, ranging up to 20 years (table 2). In the adult centres, there was no relationship between symptom duration and age at time of assessment (figure 5), but there was a direct relationship apparent in some paediatric 


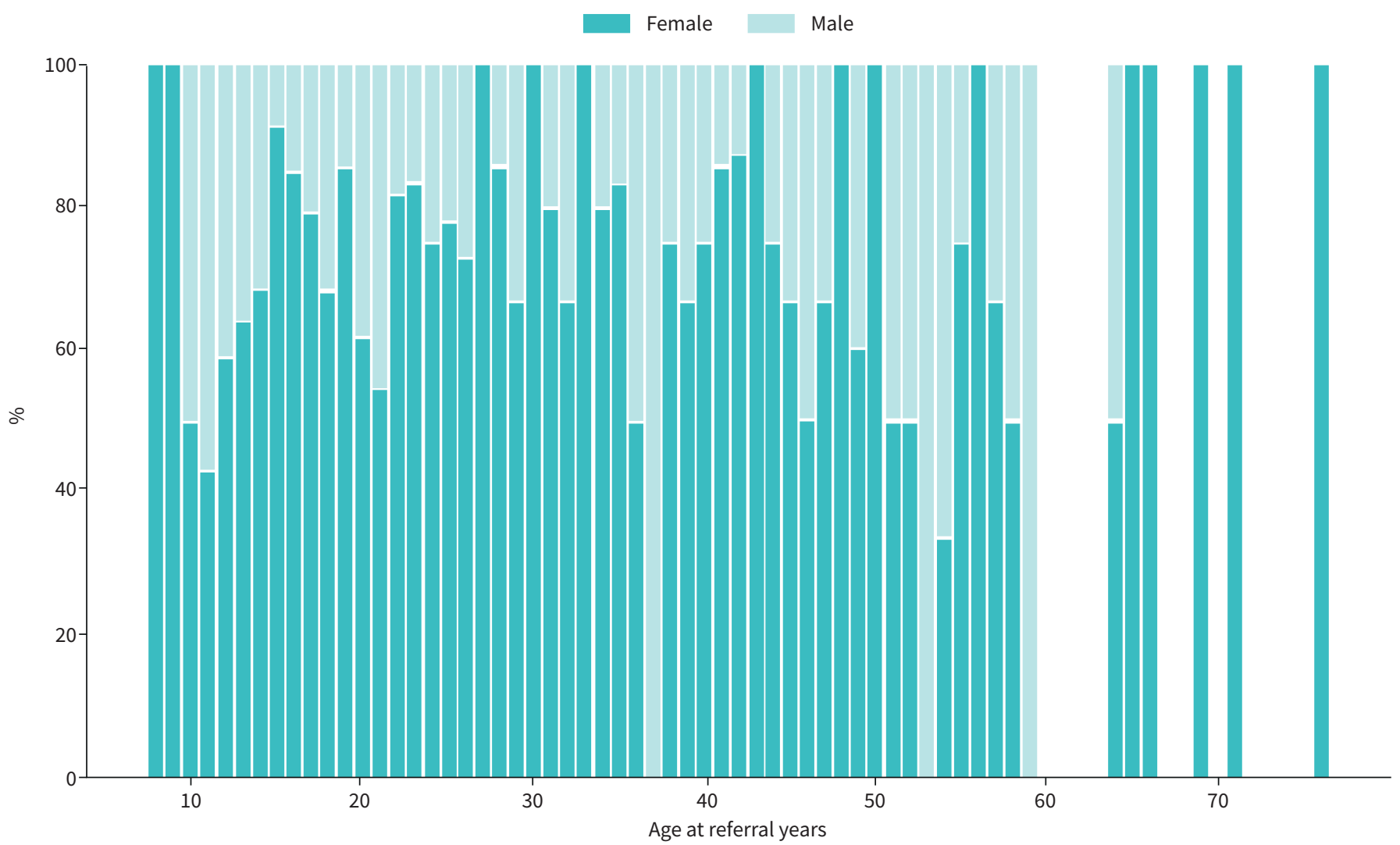

FIGURE 3 Overall sex distribution by age for individuals with confirmed exercise-induced laryngeal obstruction.

populations: Cayuga Medical Center (Spearman's rho 0.35; $\mathrm{p}=0.01$ ) and at Bispebjerg Hospital (Spearman's rho 0.19; $\mathrm{p}=0.01$ ).

Interventions for EILO varied between centres (figure 6); all centres appear to employ most treatment modalities, although the use of individual modalities varied considerably. Notably, speech and language therapy was the most frequently reported intervention employed in $44 \%$ of individuals with EILO overall.

\section{Discussion}

This multicentre collaboration provides the first international perspective on the key characteristics of EILO. Our analysis of over a thousand patients referred to expert respiratory centres confirms that in globally distinct regions, individuals with EILO appear to share similar demographic characteristics.

\begin{tabular}{|c|c|c|c|c|}
\hline & Total & EILO & No EILO & p-value \\
\hline Subjects $n$ & 1007 & 586 & 421 & \\
\hline Age years & $24(8-76)$ & $21(8-76)$ & $27.5(8-75)$ & $<0.001$ \\
\hline Female/male n ( $\%$ female) & 713/295 (71) & 444/142 (76) & 269/152 (64) & $<0.001$ \\
\hline Body mass index $\mathrm{kg} \cdot \mathrm{m}^{-2}$ & $22.8 \pm 15.1$ & $22.2 \pm 14.9$ & $23.9 \pm 15.7$ & 0.08 \\
\hline Asthma & 398 (39) & $213(36)$ & $185(44)$ & 0.02 \\
\hline \multicolumn{5}{|l|}{ Athlete status } \\
\hline Non-competitive & $451(45)$ & $239(41)$ & $212(50)$ & $<0.001$ \\
\hline Competitive & $267(26)$ & 192 (33) & 75 (18) & \\
\hline National/international level & $158(16)$ & $91(16)$ & $67(16)$ & \\
\hline Unclassifiable & 133 (13) & $64(11)$ & $67(16)$ & \\
\hline
\end{tabular}


TABLE 3 Demographics of individuals with exercise-induced laryngeal obstruction (EILO)

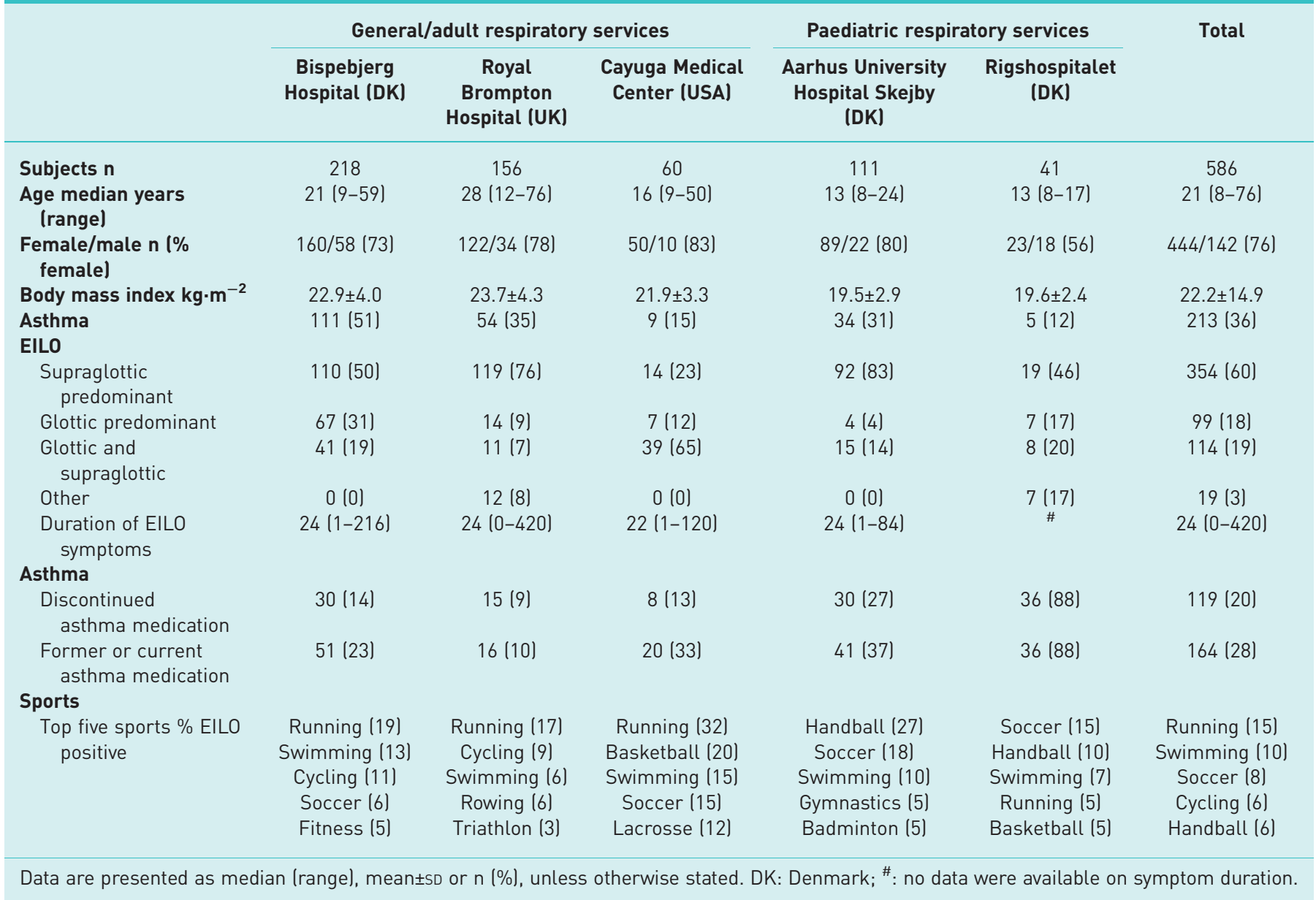

Specifically, EILO was most frequently diagnosed in young individuals in both paediatric and adult centres, and appears to exhibit a female preponderance, when compared to those without EILO (76\% and $64 \%$, respectively; $\mathrm{p}<0.001$ ). Our analyses also highlight consistency across differing healthcare systems, especially in terms of an apparent consistent delay to diagnosis (i.e. 2 years on average) and high rate of inappropriate treatment prescription, with one-fifth prescribed asthma therapy despite lack of objective evidence of asthma.

A further important similarity across the different centres was that supraglottic level EILO (i.e. arytenoid structures and not the vocal cords) was the most common CLE finding (60\%). This challenges use of the nomenclature "exercise-induced vocal cord dysfunction", given the closure does not often occur at the vocal cord level, strengthening the case for the term EILO to be more widely adopted internationally [12].

\section{Characteristics of individuals diagnosed with EILO}

Although the majority of individuals with EILO in the present study were adolescent or young adults, consistent with previous single-centre reports, there was a notable proportion of individuals referred and ultimately diagnosed with EILO in middle-age or older (figure 2). Interestingly, advancing age did not appear to relate to symptom duration in adults (figure 5); this observation might indicate that EILO does not necessarily always arise from an innate anatomical/structural/genetic factor, but could also be acquired.

The observed female predominance is consistent with previous reports $[2,4,13]$. It has been speculated that the laryngeal growth difference between genders seen in the peri-pubertal age group might explain this observation $[2,14]$, but some studies describing paediatric populations report a more equal gender distribution [3]. Although the paediatric centres involved in the current study included individuals from 8 years of age, the majority of paediatric patients were teenagers, limiting our ability to further evaluate this issue. There was however a notable difference in the gender distribution at the two Danish paediatric centres, i.e. Aarhus University Hospital Skejby ( $80 \%$ female) and Rigshospitalet (56\% female). It is also 
a)

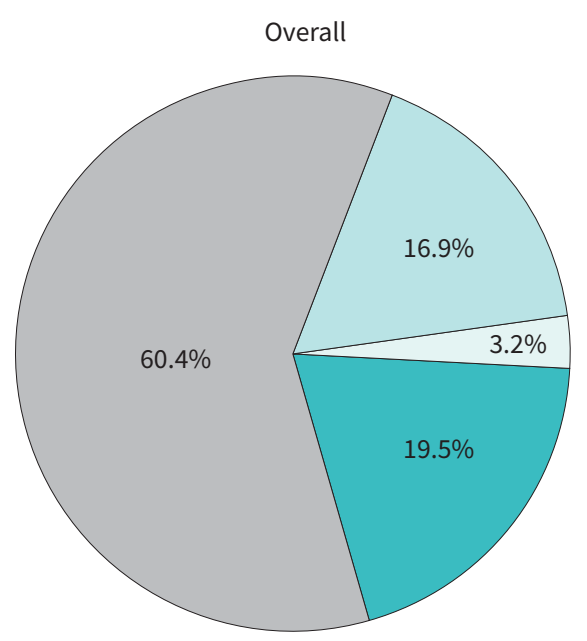

Bispebjerg

Hospital (DK)
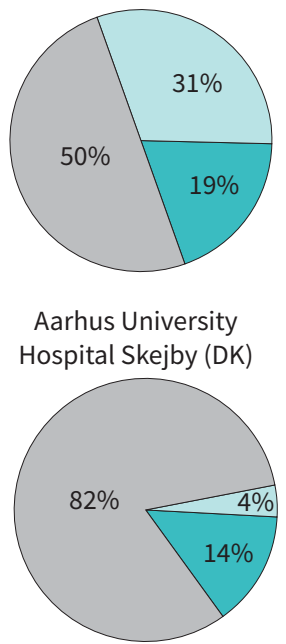

Royal Brompton

Hospital (UK)

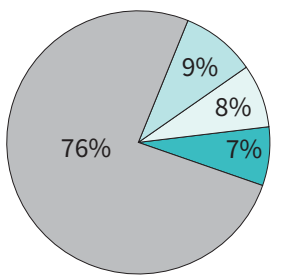

Rigshospitalet (DK)

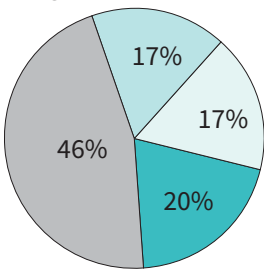

Cayuga

Medical Center (USA)

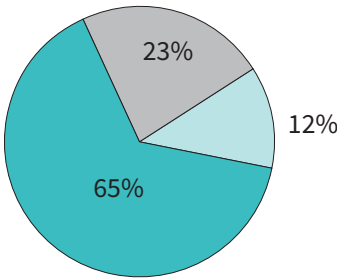

Unclassifiable

Glottic predominant

Glottic and supraglottic equally predominant

Supraglottic predominant

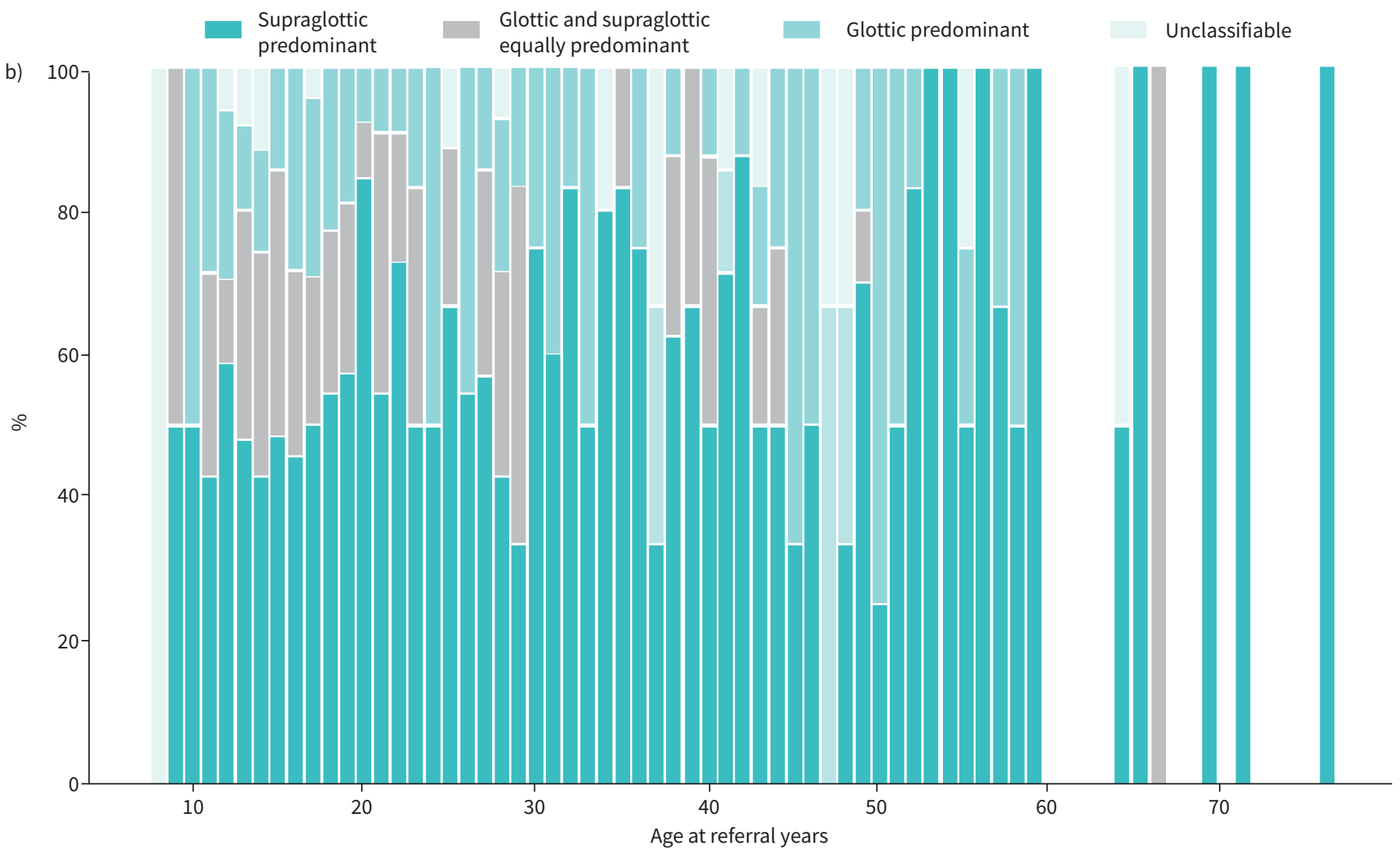

FIGURE 4 a) Exercise-induced laryngeal obstruction (EILO) subtype overall and by centre. b) EILO subtypes by age, overall. DK: Denmark.

noteworthy that the gender distribution of all referred individuals closely matches that of individuals with EILO (tables 1 and 2), and thus the gender differences observed may be explained by referral pattern.

The majority of studies, to date, have focused on addressing EILO in competitive athletes. In contrast, in the current study over half of all those tested did not describe themselves as competitive, i.e. they were only partaking in recreational sports. Across the whole cohort EILO was found in individuals partaking in a wide variety of sports, but endurance/high-intensity sports (e.g. running, swimming, cycling) were most frequently reported, probably relating to the known influence of high and prolonged ventilatory demand on the respiratory system [15]. This aligns with studies reporting a high prevalence of EILO in recreational 

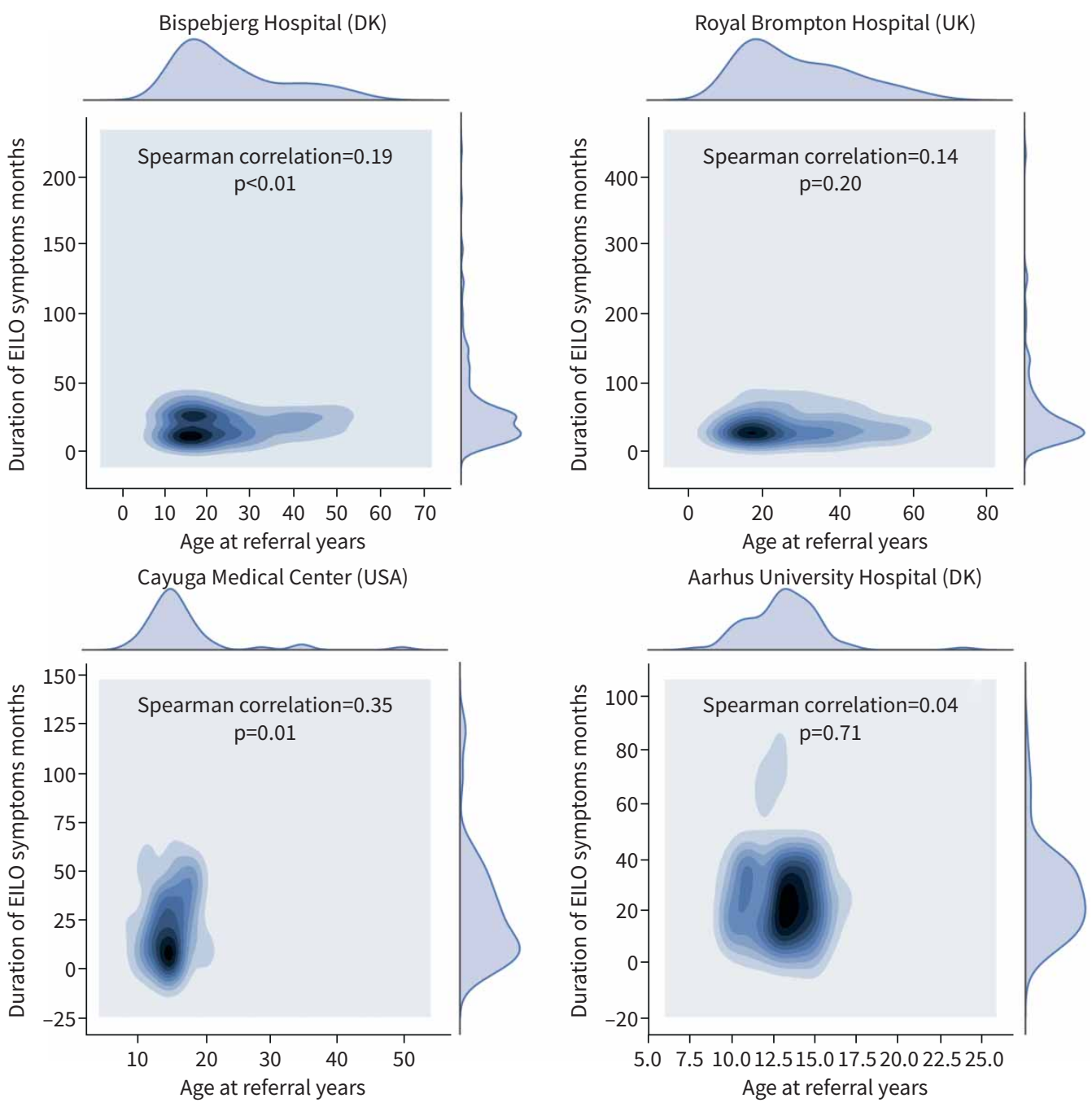

FIGURE 5 Kernel density estimation plots depicting the distribution of age and symptom duration in individuals with exercise-induced laryngeal obstruction (EILO) by centre. Symptom duration data from Rigshospitalet, Denmark (DK) were not available.

FIGURE 6 Treatment approaches utilised for exercise-induced laryngeal obstruction. Exercise: physical exercise to improve fitness encouraged/prescribed.
Overall

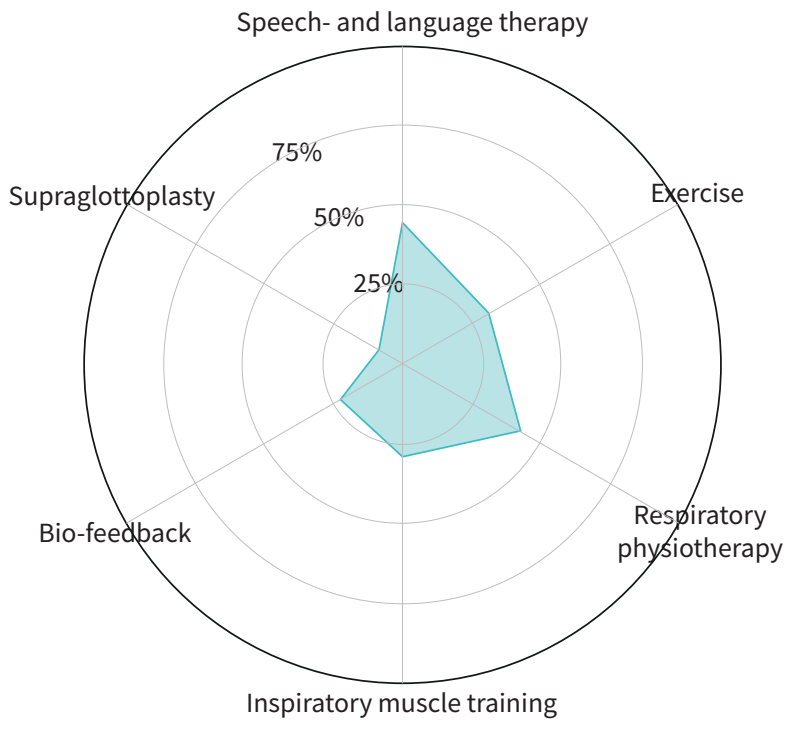


runners partaking in the Park Run [16]. Olin et al. [17] has also previously shown that the severity of laryngeal obstruction in EILO is related to exercise intensity, but it is currently unclear if chronic and repeated exposure to a heightened ventilatory load is associated or indeed a pre-requisite to the development of EILO. Regardless, the current findings in both competitive and non-competitive physically active individuals have more broad-reaching implications regarding the impact of EILO on general ability to partake in and enjoy physical activity. Indeed, this does not appear to be a condition confined exclusively to highly trained or elite sports people. Improved recognition and diagnosis of EILO in this context is therefore important to mitigate against the impact of EILO on exercise avoidance and potentially sedentary lifestyle behaviour [6].

The subclassification of CLE findings varied across centres (table 3). Although all centres utilised a standardised scoring system in combination with clinically compatible features to confirm diagnosis, an improved scoring system and a uniform diagnostic approach are warranted [18].

\section{Comorbidities of EILO}

Our analysis reveals that a significant overlap is apparent between asthma and EILO, in line with previous studies $[3,4,19]$. It is also consistent with a recent meta-analysis indicating that some form of paroxysmal laryngeal closure is likely to be apparent in at least one in four people with asthma [20]. The reasons for this overlap are currently unclear but may relate to common neural or inflammatory pathways simultaneously affecting both the lower and upper airways. This is speculative, and at present our understanding of the pathophysiology and risk factors underpinning EILO is poor. There are currently no robust data describing any distinct laryngeal pathophysiology in individuals with EILO, and there is a need to further understand the relationship between EILO and asthma, to promote improved management.

In contrast, more than one-quarter of individuals with EILO were found to have been prescribed asthma therapy with no evidence of a diagnosis of asthma, i.e. EILO had been a mimic of asthma leading to misdiagnosis. Individuals found to have EILO but no evidence of asthma had been prescribed asthma therapy for a median symptom duration of 2 years and in some individuals as long as 20 years (table 3 ). We found that medication could be discontinued in $20 \%$ of all individuals with EILO, suggesting that individuals were taking asthma medication for the entire duration of their respiratory symptoms. This amounts to a cumulative total of 308 patient-years of unnecessary asthma treatment given, or 3.7 years per patient on average (excluding Rigshospitalet where these data were not available).

We found a low proportion of individuals with reflux (3\% of confirmed EILO). This is in contrast to some data on vocal cord dysfunction (VCD)/glottic EILO that suggest an association between reflux and VCD. This discrepancy may be explained by the retrospective nature of this study, as well as the predominance of supraglottic EILO.

\section{Treatment approach}

There are currently no robust prospective randomised controlled trials addressing treatment of EILO and thus no consensus regarding the best approach to management. This is the likely explanation for the apparent disparity in therapeutic approaches adopted by different centres (figure 6). Most centres favoured a multidisciplinary team-based approach to treatment and offered either speech and language therapy or respiratory physiotherapy $[8,21]$.

\section{Methodological considerations}

Our study design was dictated by a pragmatic retrospective data entry process. It is therefore likely to be exposed to the limitations inherent to inaccurate data capture and selection/referral bias and thus not useful for estimating the prevalence of EILO. To mitigate against this risk, we required centres to record their data in a standardised template, but despite this approach, we recognise that some data were incomplete. We mandated that all EILO diagnoses were confirmed with the gold standard diagnostic test of CLE testing, and this diagnosis was confirmed by experienced clinicians, utilising the currently most widely employed scoring system [10]. We are careful not to generalise findings to populations outside of those patients referred to and assessed by specialist centres; the true prevalence of EILO in the general population is likely lower than our data suggest, as previously reported by CHRISTENSEN et al. [5] and JoHANSSON et al. [3]. The report highlights that CLE testing is a well-tolerated test that can be performed in children as young as 8 years of age.

A diagnosis of asthma was based on the local standard-of-care diagnostic workup. We did not record detailed information on how the diagnosis of asthma was obtained; however, all participating centres are specialist asthma centres and employ objective testing to confirm or refute a diagnosis of asthma. Owing to the detail of the available data, we were unable to distinguish between asthma with EIB and asthma alone; however, all centres used eucapnic voluntary hyperventilation $(\mathrm{EVH})$ challenge testing or exercise 
challenge testing to diagnose EIB. We did not quantify the amount of treatment (e.g. number of therapy sessions) given and the treatment outcome, as this information was not available in most of the participating centres. Prospective intervention studies with valid outcome measures and a structured assessment of symptoms using validated questionnaires are needed to develop diagnostic algorithms and assess treatment efficacy for EILO.

In conclusion, the findings from this study indicate that individuals with EILO exhibit similar demographic characteristics despite presenting with symptoms in globally distinct regions. It appears to be most frequently diagnosed in adolescence with a slight female predominance, although a small proportion of individuals appear to develop EILO in later life. Individuals with EILO are frequently misdiagnosed and thus inappropriately prescribed asthma inhalers while continuing to suffer from symptoms that limit their ability to exercise or maintain an active lifestyle. More work is needed now not only to understand the underlying risk factors and pathophysiology, but also to undertake prospective multicentre randomised controlled trial studies to evaluate the best approach to treatment.

Author contributions: All authors played a role in the intellectual content and writing of the manuscript. In addition: J.H. Hull and E.S. Walsted conceived and designed the study; S.L. Rubak, L. Pedersen and A. Getzin substantially contributed to the original idea, design and interpretation; E.S. Walsted, J.H. Hull, B. Famokunwa, L. Anderson, V. Backer, L. Pedersen, F. Buchval and A. Getzin were involved in data collection; and E.S. Walsted prepared the data for presentation and prepared the manuscript in collaboration with J.H. Hull. All authors critically revised the manuscript for important intellectual content and approved the final version.

Conflict of interest: None declared.

\section{References}

1 Nordang L, Norlander K, Walsted ES. Exercise-induced laryngeal obstruction - an overview. Immunol Allergy Clin 2018; 38: 271-280.

2 Halvorsen T, Walsted ES, Bucca C, et al. Inducible laryngeal obstruction: an official joint European Respiratory Society and European Laryngological Society statement. Eur Respir J 2017; 50: 1602221.

3 Johansson H, Norlander K, Berglund L, et al. Prevalence of exercise-induced bronchoconstriction and exercise-induced laryngeal obstruction in a general adolescent population. Thorax 2015; 70: 57-63.

4 Nielsen EW, Hull JH, Backer V. High prevalence of exercise-induced laryngeal obstruction in athletes. Medicine Sci Sports Exerc 2013; 45: 2030-2035.

5 Christensen PM, Thomsen SF, Rasmussen N, et al. Exercise-induced laryngeal obstructions: prevalence and symptoms in the general public. Eur Arch Oto-rhino-l 2011; 268: 1313-1319.

6 Maat RC, Hilland M, Røksund OD, et al. Exercise-induced laryngeal obstruction: natural history and effect of surgical treatment. Eur Arch Oto-rhino-l 2011; 268: 1485-1492.

7 Maat RC, Roksund OD, Olofsson J, et al. Surgical treatment of exercise-induced laryngeal dysfunction. Eur Arch Oto-rhino-l 2007; 264: 401-407.

8 Olin JT, Deardorff EH, Fan EM, et al. Therapeutic laryngoscopy during exercise: a novel non-surgical therapy for refractory EILO. Pediatr Pulm 2017; 52: 813-819.

9 Heimdal J-H, Roksund OD, Halvorsen T, et al. Continuous laryngoscopy exercise test: a method for visualizing laryngeal dysfunction during exercise. Laryngoscope 2006; 116: 52-57.

10 Maat RC, Røksund OD, Halvorsen T, et al. Audiovisual assessment of exercise-induced laryngeal obstruction: reliability and validity of observations. Eur Arch Oto-rhino-l 2009; 266: 1929-1936.

11 McGrath S, Zhao X, Zhen Z, et al. One-sample aggregate data meta-analysis of medians. Stats Med 2018; 38: 969-984.

12 Christensen PM, Heimdal J-H, Christopher KL, et al. Obstructions ETF on IL. ERS/ELS/ACCP 2013 international consensus conference nomenclature on inducible laryngeal obstructions. Eur Respir Rev 2015; 24: 445-450.

13 Buchvald F, Phillipsen LD, Hjuler T, et al. Exercise-induced inspiratory symptoms in school children. Pediatr Pulm 2016; 51: 1200-1205.

14 Wysocki J, Kielska E, Orszulak P, et al. Measurements of pre- and postpubertal human larynx: a cadaver study. Surg Radiol Anat 2008; 30: 191-199.

15 Kippelen P, Anderson SD, Hallstrand TS. Mechanisms and biomarkers of exercise-induced bronchoconstriction. Immunol Allergy Clin North Am 2018; 38: 165-182.

16 Sails J, Hull JH, Allen $\mathrm{H}$, et al. High prevalence of exercise-induced stridor during Parkrun: a cross-sectional field-based evaluation. BMJ Open Respir Res 2020; 7: e000618.

17 Olin JT, Clary MS, Fan EM, et al. Continuous laryngoscopy quantitates laryngeal behaviour in exercise and recovery. Eur Respir J 2016; 48: 1192-1200.

18 Walsted ES, Hull JH, Hvedstrup J, et al. Validity and reliability of grade scoring in the diagnosis of exercise-induced laryngeal obstruction. ERJ Open Res 2017; 3: 00070-2017.

19 Rundell KW, Jenkinson DM. Exercise-induced bronchospasm in the elite athlete. Sports Med 2002; 32: 583-600.

20 Lee J-H, An J, Won H-K, et al. Prevalence and impact of comorbid laryngeal dysfunction in asthma: a systematic review and meta-analysis. J Allergy Clin Immun 2020; 145: 1165-1173.

21 Shaffer M, Litts JK, Nauman E, et al. Speech-language pathology as a primary treatment for exercise-induced laryngeal obstruction. Immunol Allergy Clin 2018; 38: 293-302. 